\title{
Lymphocyte glycogen content in various diseases
}

\author{
R. VAUGHAN JONES, G. P. GOFFI, AND M. S. R. HUTt \\ From the Louis Jenner Laboratory, St. Thomas's Hospital and Medical School, London
}

SYNOPSIS The lymphocyte glycogen content has been measured semi-quantitatively by staining peripheral blood films by the periodic-acid-Schiff (P.A.S.) technique. The high values reported in chronic lymphatic leukaemia, lymphosarcoma, Hodgkin's disease, and infectious mononucleosis have been confirmed. High values have also been found in cases with chronic suppuration, ulcerative colitis, myelomatosis, and epithelial malignancies. Our results suggest that a high lymphocyte glycogen content may be produced by several different mechanisms and that the test is of little value in differential diagnosis.

An increased glycogen content in the lymphocytes of the peripheral blood has been reported in a number of diseases affecting the lymphoid system; these include chronic and subacute lymphatic leukaemia, lymphosarcoma, Hodgkin's disease, and some cases of infectious mononucleosis (Wislocki, Rheingold, and Dempsey, 1949; Astaldi and Verga, 1957; Mitus, Bergna, Mednicoff, and Dameshek, 1958; and Quaglino and Hayhoe, 1959). However, apart from occasional observations on patients with other blood disorders there are no reports on the lymphocyte glycogen content in diseases not primarily affecting the lymphoid system. In the present study we have estimated the lymphocyte glycogen content in a random sample of hospital patients suffering from a variety of disorders. We have also selected certain particular groups of patients, such as those with malignant epithelial tumours or chronic infections, in the hope that light might be thrown on the significance of a high lymphocyte glycogen content.

\section{METHODS}

Films of peripheral blood were air dried and fixed in methyl alcohol. Staining was by a modification of the P.A.S. technique described by Quaglino and Hayhoe (1959). After being rinsed in distilled water the films were oxidized in $0.5 \%$ aqueous periodic acid for 10 minutes and then stained for $\mathbf{3 0}$ minutes in a mixture of five parts of 'cold' Schiff reagent (after Lillie) and one part of $0.5 \%$ aqueous periodic acid. After 10 minutes in running tap water they were counterstained in 1 in 4,000 aqueous haematoxylin (Mallory) for 10 minutes, washed in running tap water for five minutes, dried, cleared in xylol and mounted.

Received for publication 6 May 1961.
The stained slides were examined by two observers independently. Fifty lymphocytes were counted by each of the two observers. Since many of the cases had normal or low lymphocyte counts, it was found impracticable for each observer to count 100 cells. The final score was obtained by addition of these separate totals. Monocytes and leucoblasts were not included in the counts. In the majority of patients the scores given are the result of observations on a single blood film. In several of the patients with malignant lymphomata serial observations were made and the score quoted is the mean of these.

\section{SCORING}

The scoring was based on the method of Mitus et al. (1958).

Cells with no P.A.S.-positive granules. . . . . . . . . . 0

Cells with less than 9 P.A.S.-positive granules.......1

Cells with over 9 P.A.S.-positive granules but no large

clumps.................................2

Cells with three or more concentric rings of P.A.S.positive granules or with large clumps of P.A.S.positive material ...........................

\section{MATERIAL}

Blood films were studied from 31 hospital patients with miscellaneous disorders, 30 patients with malignant epithelial tumours, 17 patients with lymphosarcoma, 13 patients with chronic lymphatic leukaemia, 22 patients with Hodgkin's disease, nine patients with infectious mononucleosis, eight patients with chronic suppuration, and four with acute pyogenic infections. In addition, small numbers of other specific diseases were studied.

RESULTS

MISCELLANEOUS DISORDERS (CONTROLS) The 31 patients in this group were separated clinically 


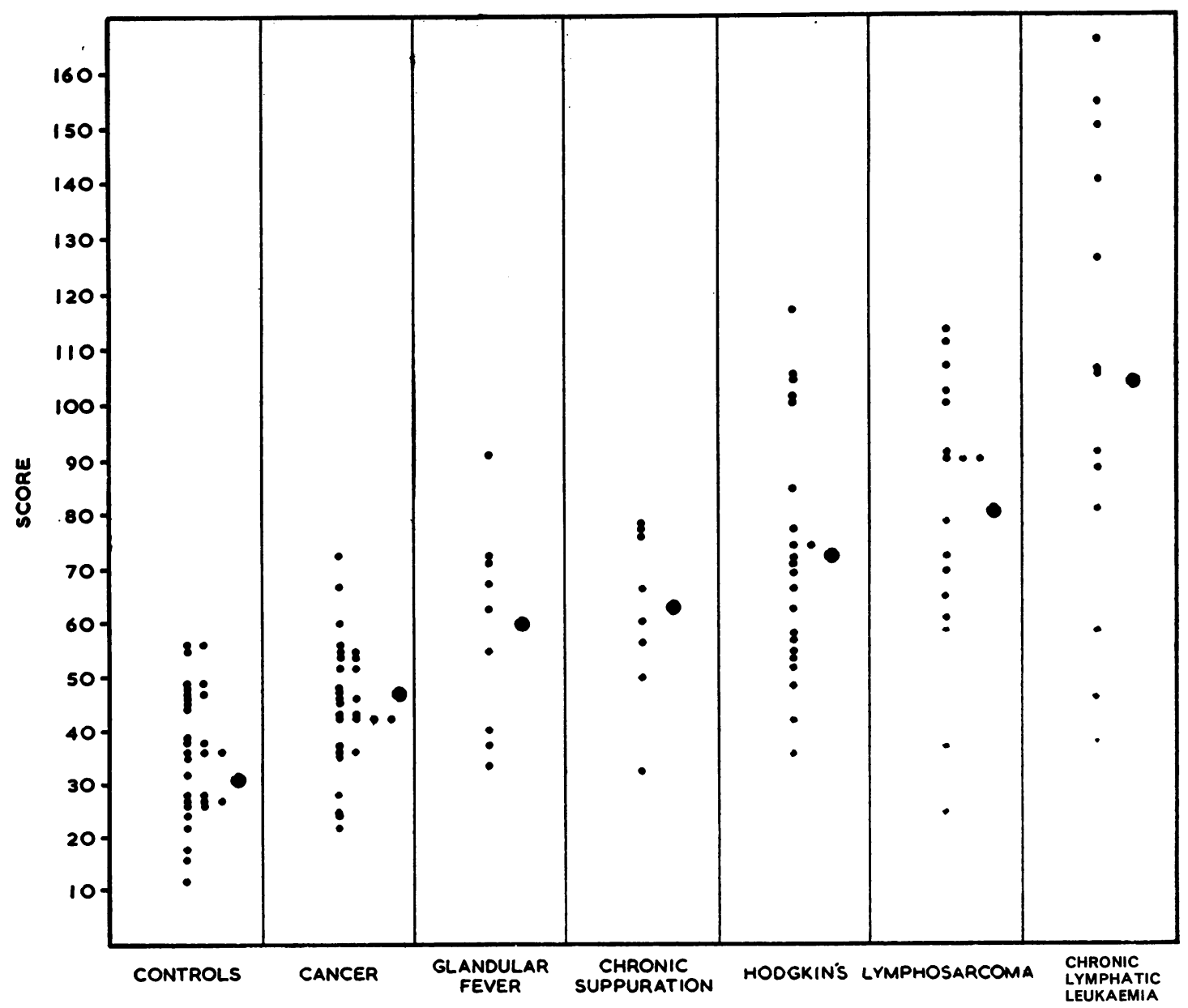

FIG. 1. The P.A.S. score in various groups of diseases. Each point represents one patient. The mean value is indicated by the larger points.

because they had no evidence of acute inflammation, chronic sepsis, carcinoma, or disorders of the reticuloendothelial system. They included patients with disorders such as coronary thrombosis, cardiac failure, hypertension, and diabetes. The range of scores in this group was from 12 to 56 with a mean of 31 . These figures correspond very closely to the mean score in normal individuals given by Quaglino and Hayhoe (1959).

LYMPHOSARCOMA Seventeen patients with this condition were studied and showed scores ranging from 24 to 111 with a mean of 80 . As can be seen in Fig. 1, only two patients had scores below 50 . One of these was clinically free from any evidence of activity but the other had widespread disease. Some of the patients with high scores were clinically active and others quiescent. We have not had a sufficient number of serial observations to assess the effects of treatment on the score. A high score did not, however, appear to be related to an absolute lymphocytosis, the presence of abnormal lymphocytes, or the severity of the disease.

CHRONIC LYMPHATIC LeUKAEMIA The highest total scores were obtained in this condition with a range of 37 to 168 and a mean of 104. There was no general relationship between the scores and the total white cell count or clinical state of the patient.

HODGKIN's DISEASE Twenty-two patients were studied and were found to have a range of 35 to 117 
with a mean score of 71 . High scores were found in some patients without evidence of clinical activity. A number of patients with Hodgkin's disease had to be excluded because they had very low lymphocyte counts either as a result of the disease or of treatment. In several of these cases it was noted that the few lymphocytes that could be seen had a very high glycogen content. Four patients with reticulum cell sarcoma were studied. These showed scores of 28 to 99 with a mean of 56 .

MALIGNANT EPITHELIAL NEOPLASMS Thirty patients were studied with a variety of epithelial neoplasms. They showed scores ranging from 24 to 76 with a mean of 47. Although there was considerable overlap with the control series the mean score was higher. In order to see whether these figures bear any relationship to the type of neoplasm we have broken down the figures as follows:- Breast (six cases), range 42 to 51 , mean 49; cervix (four cases), range 43 to 76 , mean 58 ; ovary (seven cases) range 24 to 55, mean 39; bladder (three cases), range 42 to 72 , mean 57; bronchus (four cases), range 22 to 60 , mean 45; others (six cases), range 28 to 55, mean 41 .

No significant relationship exists between the type of neoplasm and the scores, and as far as we could assess there was no relationship between the extent of the tumour and a high score. One of the patients with bronchial carcinoma had an initially low score; a repeat examination in this case several weeks later showed a high score but on this occasion the patient showed evidence of a superimposed infection.

ACUTE PYogenic inflammation. None of the four patients studied showed a high score. The range was 14 to 39 with a mean of 28 .

CHRONIC SUPPURATION Eight patients with chronic suppurative lesions were studied. These included patients with a lung abscess, a perinephric abscess, chronic osteomyelitis, and a chronic empyema. The range of scores in this group was 32 to 78 with a mean of 62. Three patients with chronic ulcerative colitis who might be classified under chronic suppuration showed a range of 66 to 91 .

INFECTIOUS MONONUCLEOSIS Nine patients were studied with a range of 33 to 91 and a mean of 59; six of the nine had counts of 55 or above.

OTHER DISORDERS Four patients with myelomatosis all showed high scores. The range was 67 to 90 with a mean of 77. None of these cases showed plasma cells in the peripheral blood. Scores of over 70 were also obtained from one of three cases of aplastic anaemia and one case of auto-immune haemolytic anaemia.

\section{DISCUSSION}

These results show that an increased amount of $\frac{C}{\partial}$ glycogen in the lymphocytes may occur in disorders which do not primarily affect the lymphoid system, such as carcinoma and chronic suppuration. Although in this series a score of over 100 was only found in patients with chronic lymphatic leukaemia, lymphosarcoma, or Hodgkin's disease, it is evident that the level of lymphocyte glycogen is of little value as a differential diagnostic test. This point was emphasized by our experience with one patient who was admitted with a pyrexia of unknown origin diagnosed as possible Hodgkin's disease; she had a score of 80 , which was thought to be good confirmatory evidence of a malignant lymphoma, but was later found to have a chronic perinephric abcess.

It remains to ask whether these results throw any light on the mechanism underlying a high lymphocyte glycogen content. It has been suggested that the high scores in chronic lymphatic leukaemia and lymphosarcoma may be due to an alteration of metabolism consequent on some fundamental 'neoplastic' change in the cell (Astaldi and Verga, 1957). Recently, however, Quaglino and Hayhoe (1959) have reported high scores in Hodgkin's disease and infectious mononucleosis. Although Hodgkin's disease is usually considered as a neoplastic disease of the lymphoid system there is no direct evidence that the lymphocytes themselves undergo a fundamental change analogous to that occurring in lymphosarcoma. Quaglino and Hayhoe (1959) suggest that the high glycogen content in Hodgkin's disease and infectious mononucleosis may have a basically different cause from that which operates in lymphatic leukaemia. A possible analogous situation might be the high alkaline phosphatase found in the polymorphonuclear leucocytes in Hodgkin's disease and acute infections (Hayhoe and Quaglino, 1958).

A consideration of the high scores found in chronic $N$ suppuration and ulcerative colitis shows that a high lymphocyte glycogen may occur in diseases where it $N$ can be presumed that normal lymphocytes are being $\omega$ produced from the lymphoid system. It is reasonable to surmise that the high scores in these cases may be $\stackrel{0}{ }$ the result of alterations in the functional activity of $\Phi$ the lymphocyte due to activities such as antibody? formation.

Although a superadded infection may account for $\stackrel{0}{\mathbb{D}}$ the high scores in a few of the patients with car- $\frac{?}{\Phi}$ cinoma, many of the patients with high scores had $\varrho$ no evidence of infection. It is known that there is 
frequently a lymphocytic infiltration in the tissues adjacent to a carcinoma and it is possible that this represents a defensive mechanism analogous to that occurring in chronic inflammation. The high scores in non-infected cases of cancer might be the result of an alteration in lymphocyte metabolism associated with this reaction.
REFERENCES

Astaldi, G., and Verga, L. (1957). Acta haemat. (Basel.), 17, 129. Hayhoe, F. G. J., and Quaglino, D. (1958). Brit. J. Haemat., 4, 375.

Mitus, W. J., Bergna, L. J., Mednicoff, I. B., and Dameshek, W. (1958). Blood, 13, 748.

Quaglino, and Hayhoe, F. G. J. (1959). J. Path. Bact., 78, 521.

Wislocki, G. B., Rheingold, J. J., and Dempsey, E. W. (1949). Blood, 4, 563.

\section{Broadsheets prepared by the Association of Clinical Pathologists}

The following broadsheets (new series) are published by the Association of Clinical Pathologists. They may be obtained from Dr. R. B. H. Tierney, Pathological Laboratory, Boutport Street, Barnstaple, N. Devon. The prices include postage, but air mail will be charged extra.

3 The Detection of Barbiturates in Blood, Cerebrospinal Fluid, Urine, and Stomach Contents. 1953. L. C. NICKOLLS. 1s.

4 The Estimation of Carbon Monoxide in Blood. 1953. D. A. STANLEY. $1 \mathrm{~s}$.

5 The Identification of Reducing Substances in Urine by Partition Chromatography on Paper. 1953. G. B. MANNING. 1s.

6 The Paul-Bunnell Test. 1954. R. H. A. SWain. 1s.

7 The Papanicolaou Technique for the Detection of Malignant Cells in Sputum. 1955. F. HAMPSON. 1s.

10 Mycological Techniques: (1) Collection of Specimens. 1956. R. W. RIDDELL. 1s.

11 Mycological Techniques: (2) Cultural Isolation. 1956. R. W. RIDDELL. 1s.

12 Techniques for Demonstrating L.E. Cells. 1956. J. V. DACIE and L. S. SACKER. $1 \mathrm{~s}$.

13 The Identification of Serotypes of Escherichia coli Associated with Infantile Gastro-enteritis. 1956. JOAN TAYLOR. $1 \mathrm{~s}$.

14 The Determination of Serum Iron and Serum Unsaturated Iron-binding Capacity. 1956. ARTHUR JORDAN. 1s.

16 Preservation of Pathological Museum Specimens. 1957. L. W. PROGER. 1s.

17 Cultural Diagnosis of Whooping-cough. 1957. B. W. LACEY. $1 \mathrm{~s}$.

18 The Rose-Waaler Test. 1957. c. L. GREENBURY. 1s.

19 The Laboratory Diagnosis of Fibrinogen Deficiency. 1958. R. M. HARDISTY. 1s.
20 Investigation of Porphyrin/Porphyria. 1958. c. RIMINGTON. $1 \mathrm{~s}$.

21 Quantitative Determination of Porphobilinogen and Porphyrins in Urine and Faeces. 1958. C. RIMINGTON. $1 \mathrm{s.}$

22 Investigation of Haemolytic Anaemia. 1959. J. G. SELWYN. 1s.

23 The Dried Disc Technique for Bacterial Sensitivity Tests. 1959. R. W. FAIRBROTHER and J. C. SHERRIS. 1s.

24 Safe Handling of Radioactive Tissues in the Laboratory and Post-mortem Room. 1959. R. C. CURRAN. $1 \mathrm{~s}$.

26 The Periodic Acid-Schiff Reaction. 1959. A. G. E. PEARSE 1s.

28 Daily Fatty Acid Excretion. 1960. A. C. FRAZER. $2 s$.

29 The Preparation of Bone for Diagnostic Histology. 1960. D. H. Collins. 2 s.

31 Investigation of Haemorrhagic States with Special Reference to Defects of Coagulation of the Blood. 1961. E. K. BLACKBURN. 4s.

32 Detection of Resistance to Streptomycin, P.A.S., and Isoniazid in Tubercle Bacilli. 1961. R. CRUICKSHANK and S. M. STEWART. $2 s$.

33 The Laboratory Detection of Abnormal Haemoglobins. 1961. H. LeHMANN and J. A. M. AGER. $4 s$.

34 Titration of Antistreptolysin O. 1961. H. GOODER and R. E. O. WILLIAMS. 2s.

35 The Estimation of Faecal 'Urobilinogen.' 1961. C. H. GRAY. 2 s. 